\begin{tabular}{|l|l|}
\hline $\begin{array}{l}\text { Instituto de } \\
\text { Geriatria e Gerontologia }\end{array}$ & $\begin{array}{l}\text { Pan American Journal of Aging Research } \\
\text { PAJAR, Porto Alegre, v. 8, p. 1-8, jan.-dez. } 2020 \\
\text { ISSN-L: 2357-9641 }\end{array}$ \\
\hline http://dx.doi.org/10.15448/2357-9641.2020.1.36639 \\
\cline { 2 - 3 }
\end{tabular}

ARTIGO ORIGINAL

\title{
Perfil cognitivo e clínico psiquiátrico de idosos atendidos em um ambulatório especializado
}

\author{
Cognitive and clinical psychiatric profile of elderly served in a specialized ambulatory \\ Perfil clínico cognitivo y psiquiátrico de personas mayores servidas en un ambulatorio \\ especializado
}

Bruno Lodi ${ }^{1}$

orcid.org/0000-0003-3878-6750

brunoo_lodi@hotmail.com

\section{Paula Engroff ${ }^{1}$ \\ orcid.org/0000-0002-3639-545X \\ paula_puc@yahoo.com.br}

\section{Francisco Pascoal}

Júnior ${ }^{1}$

orcid.org/0000-0001-5827-7270

pascoaljunior@uol.com.br

\section{Guilherme Marcos}

Nogueira $^{1}$

orcid.org/0000-0002-0527-6175

guilhermeneurociencia@gmail.com

Vanessa Sgnaolin ${ }^{1}$

orcid.org/0000-0002-9914-7146 vanessa.sgnaolin@pucrs.br

\section{Alfredo Cataldo Neto ${ }^{1}$} orcid.org/0000-0002-8082-1866 cataldo@pucrs.br

Received on: Dec. $11^{\text {th }}, 2019$. Approved on: May. $6^{\text {th }}, 2020$. Published on: Sep. 17, 2020

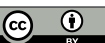

Artigo está licenciado sob forma de uma licenç Creative Commons Atribuição 4.0 Internacional.
Resumo

Objetivo: descrever o perfil cognitivo e clínico psiquiátrico de idosos provenientes da atenção primária e atendidos em um ambulatório especializado de um hospital universitário

Métodos: estudo transversal, descritivo e analítico, coletado de forma prospectiva em uma amostra da população idosa (60 anos ou mais) cadastrada na Estratégia Saúde da Familia do município de Porto Alegre e atendidos em um ambulatório especializado de um hospital universitário, no periodo de julho de 2015 a julho de 2016. As variáveis analisadas foram sociodemográficas (sexo, idade, escolaridade), clínicas (diagnóstico psiquiátrico) e avaliação cognitiva (Exame Cognitivo Addenbrooke - versão revisada, ACE-R). O protocolo de pesquisa foi aprovado pelos Comitês de Ética em Pesquisa e todos os participantes assinaram o Termo de Consentimento Livre e Esclarecido.

Resultados: foram avaliados 256 individuos com média de idade de 70,8 57,1 anos, na maioria mulheres (77,7\%) e com 4 a 7 anos de estudo (32,2\%). Desses, $54,3 \%$ apresentavam alguma alteração cognitiva e $43,4 \%$ eram classificados como tendo demência, através da avaliação do ACE-R.

Conclusões: o perfil cognitivo e clínico psiquiátrico dos participantes do estudo demostrou que, no grupo normal, os idosos eram mais jovens e com maior escolaridade e, no grupo demência, os individuos eram mais velhos, com menor escolaridade e com diagnóstico de depressão.

Palavras-chave: demência, depressão, idoso, testes de estado mental e demência.

\section{Abstract}

Aims: to describe the cognitive and psychiatric clinical profile of older adults from primary care who are seen at a specialized outpatient clinic of a university hospital. Methods: this was a cross-sectional, descriptive and analytical study, prospectively collected from a sample of the elderly population (6o years or older) enrolled in the Family Health Strategy of the city of Porto Alegre and treated at a specialized outpatient clinic of a university hospital during the study period July 2015 to July 2016. The variables analyzed were sociodemographic (gender, age, education), clinical (psychiatric diagnosis) and cognitive assessment (Addenbrooke Cognitive Examination - revised version, ACE-R). The research protocol was approved by the Research Ethics Committees and all participants signed the Informed Consent Form.

Results: 256 individuals with a mean age of $70.8 \pm 7.1$ years were evaluated, mostly women ( $77.7 \%$ ) and with 4 to 7 years of study (32.2\%). Of these $54.3 \%$ had some cognitive impairment and $43.4 \%$ were classified as having dementia through the ACE-R assessment. Conclusions: the cognitive and clinical psychiatric profile of the study participants showed that in the normal group, the elderly were younger and more educated and in the dementia group the individuals were older, less educated and diagnosed with depression.

Keywords: dementia, depression, aged, mental status and dementia tests. 
Resumen

Objetivos: describir el perfil psiquiátrico cognitivo y clínico de los ancianos de atención primaria y atendidos en una clínica ambulatoria especializada de un hospital universitario.

Métodos: este fue un estudio transversal, descriptivo y analítico, recolectado prospectivamente de una muestra de la población de edad avanzada (60 años o más) inscritos en la Estrategia de Salud Familiar de la ciudad de Porto Alegre y tratados en una clínica ambulatoria especializada de un hospital universitario durante el período de estudio. Julio de 2015 a julio de 2016. Las variables analizadas fueron sociodemográficas (género, edad, educación), clínicas (diagnóstico psiquiátrico) y evaluación cognitiva (Addenbrooke Cognitive Examination - versión revisada, ACE-R). El protocolo de investigación fue aprobado por los Comités de Ética de Investigación y todos los participantes firmaron el Formulario de Consentimiento Informado.

Resultados: se evaluaron 256 individuos con una edad media de $70.8 \pm 7.1$ años, en su mayoría mujeres $(77.7 \%)$ y con 4 a 7 años de escolaridad (32.2\%). De estos, el $54,3 \%$ tenía algún deterioro cognitivo y el $43,4 \%$ se clasificó como demencia a través de la evaluación ACE-R. Conclusiones: el perfil psiquiátrico cognitivo y clínico de los participantes del estudio mostró que en el grupo normal, los ancianos eran más jóvenes y más educados y en el grupo de demencia los individuos eran mayores, menos educados y con diagnóstico de depresión. Palabras clave: demencia, depresión, anciano, pruebas de estado mental y demencia.

\section{Introdução}

A Organização Mundial da Saúde (OMS) considera envelhecido o país em que $14 \%$ da sua população se encontra na faixa etária igual ou superior aos 65 anos. Dados do Instituto Brasileiro de Geografia e Estatística (IBGE) evidenciam que a população brasileira representa atualmente 9.5\% de idosos da população total. As projeções são de que em 2032 o Brasil atinja o percentual de 14\% da população acima dos 65 anos e, que em 2060, esse número representará $25 \%$ da população total.7

O envelhecimento da população tem despertado o interesse para estudos relacionados à prevalência e à incidência da demência na população com idade superior a 65 anos. Para a OMS a demência é uma das maiores causas de incapacidade e de dependência entre pessoas idosas no mundo. ${ }^{8} \mathrm{Em} 2015$, o custo global do tratamento de pacientes com demência no mundo foi estimado em 818 bilhões de dólares, o equivalente a $1,1 \%$ do Produto Interno Bruto (PIB) mundial, representando 0,2\% dos países de baixo e médio desenvolvimento e $1,4 \%$ do PIB dos paises desenvolvidos. Dados referem ainda que, mundialmente, cerca de 50 milhões de pessoas sofrem de demência e 10 milhões de novos casos são diagnosticados ao ano. ${ }^{9}$

Segundo uma revisão sistemática, cerca de 35,6 milhões de pessoas em todo o mundo viviam com demência em 2010. A previsão é de que esse número duplique a cada 20 anos, representando 65.7 milhões em 2030 e 115,4 milhões em 2050. ${ }^{4}$ No Brasil, estudos também apresentam resultados quanto à incidência de demência na população idosa. Um deles realizado na cidade de Porto Alegre, demonstrou uma taxa de 14,8/1000/ano em idosos acima de 65 anos para Doença de Alzheimer (DA) e outro, na cidade de Catanduva (interior de São Paulo), com incidência de 7,7/1000/ano para DA e 13,8/1000/ano para demência.5.6 Nesse último estudo, a incidência de demência dobrou a cada cinco anos, sem diferença por sexo, mas houve uma maior incidência de demência em mulheres com idade mais avançada. Também se observou uma tendência à maior incidência entre analfabetos. ${ }^{6}$

Em relação à associação de demência e de variáveis clínicas foi observado que pessoas com demência têm duas vezes mais chances de apresentar depressão do que pessoas sem demência e que o risco variava pelo tipo de demência.?

Este artigo tem como objetivo principal descrever o perfil cognitivo e clínico psiquiátrico de idosos de baixa escolaridade, provenientes da atenção primária e atendidos em um ambulatório especializado de um hospital universitário.

\section{Métodos}

\section{Delineamento}

Estudo transversal descritivo e analitico, coletado de forma prospectiva em uma amostra da população idosa (60 anos ou mais) cadastrada na Estratégia Saúde da Família (ESF) do município de Porto Alegre, atendidos em um ambulatório interdisciplinar especializado em geriatria psiquiátrica de um hospital universitário.

\section{Amostra estudada}

Um total de 346 indivíduos foram avaliados e 256 foram incluidos na amostra final. Todos 
os indivíduos com necessidades clinicas foram encaminhados para consultas neurológicas e psiquiátricas especializadas no mesmo hospital.

Os critérios de inclusão foram: ter 60 anos ou mais, pertencer a ESF da área de abrangência do Hospital São Lucas da Pontifícia Universidade Católica do Rio Grande do Sul (PUCRS), estar cadastrado no Programa de Envelhecimento Cerebral (PENCE) ${ }^{8}$ e aceitar participar da pesquisa por meio da assinatura do Termo de Consentimento Livre e Esclarecido (TCLE).

Os critérios de exclusão foram: não ter realizado a avaliação pelo instrumento Exame Cognitivo Addenbrooke - versão revisada (ACE-R, do inglês Addenbrooke's Cognitive Examination - Revised) e não ter diagnóstico definido ou ter diagnóstico de alteração neurológica. A avaliação e o registro foram feitos por psiquiatras e psicólogos certificados pelo conselho com experiência em distúrbios neuropsiquiátricos.

\section{Coleta de dados}

A coleta de dados dessa pesquisa foi realizada de julho de 2015 a julho de 2016 por meio de uma parceria da Pontificia Universidade Católica do Rio Grande do Sul (PUCRS) e em colaboração com a Secretária Municipal da Saúde de Porto Alegre.

As variáveis analisadas foram sociodemográficas (sexo, idade, escolaridade), clínicas (diagnóstico psiquiátrico pelo DSM-5 e avaliação cognitiva instrumento ACE-R).

O instrumento de avaliação cognitiva ACE-R utilizado na avaliação dos participantes foi desenvolvido para avaliar de maneira breve e sensivel estágios iniciais da demência e auxiliar na diferenciação dos tipos de demência. A aplicação do instrumento ACE-R leva, em média, 16 minutos e é possivel avaliar e o pontuar os domínios: atenção/ orientação (18 pontos), memória (26 pontos), fluência (14 pontos), linguagem (26 pontos) e visoespacial (16 pontos). Sua pontuação máxima é 100 e corresponde a soma de todos os domínios. A versão revisada apresenta melhorias no âmbito da aplicação e da sensibilidade do teste, além de apresentar modificações a fim de facilitar as traduções interculturais. ${ }^{4}$ Para a avaliação dos resultados do ACE-R foram utilizados os seguintes pontos de corte: cognição normal (ACE-R acima de 70), declínio cognitivo leve (DCL) (ACE-R entre 69-65) e demência (ACE-R: abaixo de 64).9

\section{Análise estatistica}

Os dados foram analisados através do programa estatístico SPSS, versão 17. As variáveis foram descritas através de frequências, médias e desvios padrões. O teste qui-quadrado de Pearson foi empregado para testar a associação entre as variáveis categóricas. As variáveis ordinais foram comparadas pelo teste de tendência linear do qui-quadrado. Na comparação das variáveis dicotômicas com uma variável quantitativa foi utilizado o teste $t$ de Student. Os resultados foram considerados significativos quando $\mathrm{P}<0,05$.

\section{Aspectos éticos}

O protocolo de pesquisa foi aprovado pelos Comitês de Ética em Pesquisa da PUCRS e pela Secretaria Municipal de Saúde Pública de Porto Alegre, atendendo às Diretrizes e Normas Regulamentadoras em Pesquisa, conforme a Resolução 466/12 do Conselho Nacional de Saúde. ${ }^{10}$ Todos os participantes ou seus representantes legais deram consentimento informado por escrito.

\section{Resultados}

Foram avaliados 256 indivíduos com média de idade de 70,8 \pm ,12 anos, na maioria mulheres (77,7\%) e com 4 a 7 anos de estudo (32,2\%). A prevalência de alteração cognitiva foi de 54,3\% e $43,4 \%$ de demência.

Os idosos do grupo cognição normal eram mais jovens e com mais anos de estudo. Já aqueles com demência eram mais velhos, principalmente com 80 anos ou mais, e apresentavam menor escolaridade (Tabela 1). 
TABELA 1 - Avaliação do Exame cognitivo Addenbrooke - versão revisada de acordo com dados sociodemográficos.

\begin{tabular}{|c|c|c|c|c|c|}
\hline \multirow{3}{*}{ Variáveis } & \multirow{3}{*}{$\begin{array}{l}\text { Total } \\
\text { n (\%) }\end{array}$} & \multicolumn{3}{|c|}{ ACE-R } & \multirow{3}{*}{$p$} \\
\hline & & $\mathrm{CN}$ & $\mathrm{DCL}$ & DEMÊNCIA & \\
\hline & & n (\%) & n (\%) & n (\%) & \\
\hline \multicolumn{6}{|l|}{ Gênero } \\
\hline Feminino & $199(77,7)$ & $89(44,7)$ & $21(10,6)$ & $89(44,7)$ & \multirow{2}{*}{$0,707^{+}$} \\
\hline Masculino & $57(22,3)$ & $28(49,1)$ & $7(12,3)$ & $22(38,6)$ & \\
\hline \multicolumn{6}{|l|}{ Faixa etária } \\
\hline $60-69$ anos & $123(48,0)$ & $69(56,1)^{3.2}$ & $11(8,9)$ & $43(35,0)^{-2,6}$ & \multirow{3}{*}{$0,001^{\ddagger}$} \\
\hline 70-79 anos & $100(39,1)$ & $40(40,0)$ & $11(11,0)$ & $49(49,0)$ & \\
\hline 80 anos ou mais & $33(12,9)$ & $8(24,2)^{-2,7}$ & $6(18,2)$ & $19(57,6)$ & \\
\hline \multicolumn{6}{|l|}{ Escolaridade } \\
\hline Analfabeto & $25(10,7)$ & $1(4,0)$ & $3(12,0)$ & $21(84,0)$ & \multirow{4}{*}{$<0,001^{\ddagger}$} \\
\hline $1-3$ anos & $66(28,3)$ & $17(25,8)$ & $9(13,6)$ & $40(60,6)$ & \\
\hline 4-7 anos & $75(32,2)$ & $37(49,3)$ & $15(20,0)$ & $23(30,7)$ & \\
\hline 8 anos ou mais & $67(28,8)$ & $55(82,1)$ & $1(1,5)$ & $11(16,4)$ & \\
\hline TOTAL & $256(100)$ & $117(45,7)$ & $28(10,9)$ & $111(43,4)$ & \\
\hline
\end{tabular}

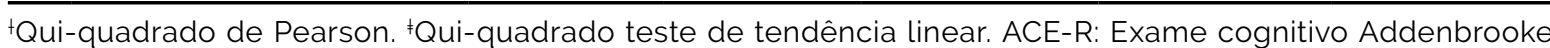
- versão revisada; CN: Cognição Normal; DCL: Declinio Cognitivo Leve.

A Tabela 2 demonstra os resultados da avaliação do ACE-R subdividindo os idosos que apresentam ou não doenças psiquiátricas. Quando estratificado o tipo de doença psiquiátrica, a depressão foi o transtorno mais frequente $(32,7 \%)$ e o que demonstrou a maior frequência de indivíduos classificados com demência (64,2\%).

TABELA 2 - Avaliação do Exame cognitivo Addenbrooke - versão revisada de acordo com o Diagnóstico Psiquiátrico.

\begin{tabular}{|c|c|c|c|c|c|}
\hline \multirow{3}{*}{ Variáveis } & \multirow{3}{*}{$\begin{array}{l}\text { Total } \\
\text { n (\%) }\end{array}$} & \multicolumn{3}{|c|}{ ACE-R } & \multirow{3}{*}{$\mathbf{p}$} \\
\hline & & $\mathrm{CN}$ & DCL & DEMÊNCIA & \\
\hline & & n (\%) & n (\%) & n (\%) & \\
\hline Sem Doença Psiquiátrica & $138(53,9)$ & $73(52,9)^{2,5}$ & $18(13,0)$ & $47(34,1)^{-3.2}$ & \multirow[b]{2}{*}{0,005} \\
\hline Doença Psiquiátrica & $118(46,1)$ & $44(37,3)^{-2,5}$ & $10(8,5)$ & $64(54,2)^{3.2}$ & \\
\hline Depressão & $67(32,7)$ & $18(26,9)^{-3.5}$ & $6(9,0)$ & $43(64,2)^{4,1}$ & $<0,001$ \\
\hline Ansiedade & $13(5,1)$ & $7(53,8)$ & $1(7,7)$ & $5(38,5)$ & 0,844 \\
\hline Transtorno de Humor Bipolar & $18(7,0)$ & $8(44,4)$ & $3(16,7)$ & $7(38,9)$ & 0,786 \\
\hline Depressão e Ansiedade & $14(5,5)$ & $7(50,0)$ & 0 & $7(50,0)$ & 0,252 \\
\hline \multirow[t]{2}{*}{ Outros Transtornos Psiquiátricos } & $6(2,3)$ & $4(66,7)$ & 0 & $2(33,3)$ & 0,610 \\
\hline & $256(100)$ & $117(45,7)$ & $28(10,9)$ & $111(43,4)$ & \\
\hline
\end{tabular}

ACE-R: Exame cognitivo Addenbrooke - versão revisada; CN: Cognição Normal; DCL: Declinio Cognitivo Leve. 
As médias dos resultados por domínios do ACE-R foram descritos na Tabela 3, levando em consideração apenas os pacientes deprimidos em relação ao grupo normal. Para todos os domínios os idosos com depressão apresentaram médias menores de resultados. O domínio visoespacial foi o único em que não foi observada diferença estatística significativa, demonstrando apenas uma tendência $(p=0,054)$.

TABELA 3 - Indivíduos normais e com depressão de acordo com a avaliação do Exame cognitivo Addenbrooke - versão revisada por domínio.

\begin{tabular}{lccc}
\hline $\begin{array}{c}\text { Dominios } \\
\text { ACE-R }\end{array}$ & $\begin{array}{c}\text { Sem } \\
\text { Depressão }\end{array}$ & $\begin{array}{c}\text { Com } \\
\text { Depressão }\end{array}$ & $\mathbf{p}$ \\
\hline $\begin{array}{l}\text { M } \pm \text { DP } \\
\text { Orienţão e }\end{array}$ & $\mathbf{M} \pm$ DP & \\
Memória & $14,70 \pm 2,78$ & $13,49 \pm 2,61$ & 0,003 \\
Fluência & $15,15 \pm 5,78$ & $12,27 \pm 5,43$ & 0,001 \\
Linguagem & $19,65 \pm 5,40$ & $17,01 \pm 5,95$ & 0,002 \\
Visoespacial & $11,69 \pm 3,83$ & $10,56 \pm 4,02$ & 0,054 \\
\hline M: média: DP: desvio padrão. & & \\
\hline
\end{tabular}

M: média; DP: desvio padrão.

\section{Discussão}

Embora preocupantemente estudos têm demonstrado que a incidência de demência está aumentando ano após ano, os medicamentos, as intervenções biomédicas e de estilo de vida ainda não obtiveram sucesso em interromper, desacelerar ou reverter o curso dessa doença. ${ }^{11}$ No presente estudo, com base em uma amostra de idosos que realizaram o teste cognitivo ACE-R, se pode destacar que individuos com idade mais avançada, baixa escolaridade e deprimidos apresentaram piores resultados na avaliação cognitiva.

Quanto mais avançada a idade maiores foram as prevalências de alteração cognitiva observadas. A variável idade representa uma das mais fortes associações com o diagnóstico de demência. Na literatura nacional e internacional são encontrados estudos com grandes populações que também demonstram essa associação. ${ }^{12,13,14}$
Alguns estudos com pacientes em idades muito avançadas, acima de 90 anos, sugerem a ocorrência de um platô, evidenciando assim que a prevalência de demência aumenta significativamente com a idade, mas não com a mesma intensidade nas faixas etárias mais avançadas. É importante também que seja desmistificado que as alterações cognitivas, por exemplo o esquecimento, fazem parte do curso natural do envelhecimento, crença popular que muitas vezes atrasa a avaliação cognitiva do idoso. Conforme o estudo de Miranda et al. ${ }^{15}$ foi observado que o tempo entre o início dos sintomas demenciais e o diagnóstico foi maior do que deveria ser, isso devido ao fato das familias e cuidadores acreditarem que os sintomas eram normais para a idade.

Outro importante achado está relacionado a escolaridade. Quanto mais anos de estudo melhores os resultados da avaliação cognitiva. Dados esses que concordam com a maioria dos estudos publicados e representam um risco seis vezes maior de demência na população brasileira com baixa escolaridade. ${ }^{16,17,18}$ Isso ocorre provavelmente porque os idosos com maior escolaridade realizam atividades diárias diversificadas e estão sujeitos a uma maior demanda cognitiva, sendo mais facilmente perceptiveis pequenas mudanças na cognição. Também é fundamental levar em consideração que esses indivíduos com maior escolaridade e, principalmente, com niveis elevados de reserva cognitiva demonstram resultados superiores de desempenho cognitivo, mesmo já tendo diagnóstico de Doença de Alzheimer. ${ }^{19}$ Provavelmente, os idosos com maior reserva cognitiva vivenciarão a sintomatologia de início da doença mais tarde. O construto da reserva cognitiva oferece uma visão positiva para abordar a crescente prevalência e incidência de demência, bem como proteger a função cognitiva ${ }^{20}$. A educação continuada e a adição de tarefas complexas, mesmo no idoso, podem ser uma intervenção para ajudar as pessoas idosas.

Os transtornos psiquiátricos estão associados com piores resultados cognitivos, principalmente nos indivíduos com diagnóstico de depressão. Os sintomas depressivos são comuns em pacientes 
idosos, sendo necessária uma boa avaliação para diferenciar sintomas depressivos sugestivos de início de um quadro demencial, pseudodemência ocorrida na depressão ou quadros sobrepostos. Aparentemente os transtornos afetivos estão relacionados com o risco maior de desenvolvimento dos quadros demenciais, o que pode ser constatado em um estudo de coorte, com 28 anos de acompanhamento. ${ }^{21}$ No curso da depressão geriátrica uma análise mostrou que o grupo com demência reversivel (pseudodemência) teve uma chance 4,69 vezes maior de desenvolver demência no seguimento do que os pacientes com

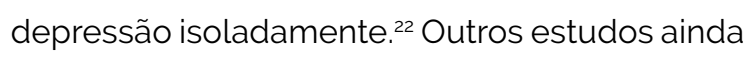
demonstraram que a prevalência de depressão pode ser de 30\% na Doença de Alzheimer e na demência vascular, e mais de $40 \%$ na demência associada às doenças de Parkinson e Huntington. ${ }^{23.24}$ Achados esses que demonstram uma relação bidirecional entre depressão e demência. Em relação à aplicação do ACE-R nos pacientes deprimidos, o estudo desenvolvido por Beckert et al. ${ }^{25}$ observou que os sintomas depressivos não modificam os valores dos testes realizados no ACE-R de idosos com baixa escolaridade.

Os idosos com depressão obtiveram pontuação média inferior em todos os domínios cognitivos avaliados quando comparados com individuos normais, com maior destaque: atenção e orientação, memória, fluência e linguagem. Considerando a hipótese que a depressão é uma doença de caráter inflamatório e de acometimento da neurotransmissão, conclui-se que possa existir um prejuizo dos domínios cognitivos, especialmente da memória. ${ }^{26} \mathrm{Em}$ muitos casos de depressão o paciente pode cursar com capacidade diminuída para pensar, concentrar-se ou tomar decisões. ${ }^{27}$ Os domínios da função executiva e da atenção, incluindo a memória são características centrais na depressão no idoso além dos sintomas de mau humor. ${ }^{28}$

As limitações do estudo são decorrentes do delineamento transversal, o que impede a avaliação da casualidade e não permite conhecer o desfecho evolutivo da população estudada. Também podemos citar a escassez de dados em relação ao DCL, explicado parcialmente pelo fato de que a busca por tratamento geralmente ocorre em estágios mais avançados da doença e pela dificuldade do diagnóstico. A força do presente estudo reside no tamanho da amostra, no rigor metodológico empregado para coleta de dados e no perfil diferenciado da casuistica composta por idosos da comunidade de baixa renda e escolaridade, fornecendo dados relevantes sobre uma condição clínica e uma população pouco estudada em nosso pais.

\section{Conclusão}

O perfil cognitivo e clínico psiquiátrico dos participantes do estudo observou que individuos com idade mais avançada, baixa escolaridade e deprimidos apresentaram piores resultados na avaliação cognitiva. É importante que os idosos, especialmente aqueles deprimidos, recebam uma avaliação cuidadosa do humor e da cognição. Em nosso país é evidente que a atenção primária à saúde não recebe a devida relevância, haja visto os escassos recursos empregados, o número limitado de consultas especializadas e os medicamentos para tratamento específico dessa população. Para que avanços na saúde pública sejam alcançados é necessário o desenvolvimento de estratégias, como medidas para diagnosticar precocemente e iniciar tratamento no momento adequado, assim minimizando os danos causados pelo atraso no diagnóstico e tratamento dos idosos com demência e depressão.

\section{Agradecimento}

O presente trabalho foi realizado com apoio da Coordenação de Aperfeiçoamento de Pessoal Nivel Superior - Brasil (CAPES) - Código de Financiamento 001.

\section{Referências}

1. Instituto Brasileiro de Geografia e Estatística. Projeção da população do Brasil e das Unidades da Federação. Brasilia: Instituto Brasileiro de Geografia e Estatística; 2019. [Capturado 2019 Abr 21]. Disponivel em: https:// www.ibge.gov.br/apps/populacao/projecao/.

2. World Health Organization. Global action plan on the public health response to dementia 2017-2025. Geneva: World Health 
Organization; 2017. [capturado 2019 Out 10]. Disponivel em: https://apps.who.int/iris/bitstream/handle/10665/259615/9789241513487-eng.pdfjjsessioni$\mathrm{d}=6 \mathrm{ED} 79 \mathrm{C2EEE}$ FFEF970C6F92127E94541B? sequence=1.

3. World Health Organization. Dementia. Geneva: World Health

Organization; 2019 [capturado 2019 Out 10]. Disponivel em: https://www.who.int/en/news-room/fact-sheets/ detail/dementia?

4. Prince M, Bryce R, Albanese E, Wimo A, Ribeiro W, Ferri CP. The global prevalence of dementia: a systematic review and metaanalysis. Alzheimers Dement. 2013:9(1):63-75. https://doi.org/10.1016/i.jalz.2012.11.007.

5. Chaves ML, Camozzato AL, Godinho C, Piazenski I, Kaye J. Incidence of Mild Cognitive Impairment and Alzheimer Disease in Southern Brazil J Geriatr Psychiatry Neurol. 2009;22(3):181-7. https://doi. org/10.1177/0891988709332942.

6. Nitrini R, Caramelli P, Herrera E, Bahia VS, Caixeta LF, Radanovic M, Anghinah R, Charchat-Fichman H, Porto CS, Carthery MT, Hartmann AP, Huang N, Smid J, Lima EP, Takada LT, Takahashi DY. Incidence of Dementia in a Community-Dwelling Brazilian Population. Alzheimer Dis Assoc Disord. 2004:18(4):241-6.

7. Andreasen P, Lönnroos E, von Euler-Chelpin MC. Prevalence of depression among older adults with dementia living in low- and middle-income countries: a cross-sectional study. Eur J Public Health. 2014:24(1):404. https://doi.org/10.1093/eurpub/ckto14.

8. Silva, AR, Sgnaolin, V, Nogueira, EL, Loureiro F, Engroff P. Gomes I. Non-communicable chronic diseases and sociodemographic associated with symptoms of depression in elderly. J Bras Psiquiatria. 2017;66(1):45-51. https://doi.org/10.1590/0047-2085000000149.

9. César KG, Yassuda MS, Porto FHG, Brucki SMD, Nitrini R. Addenbrooke's cognitive examination-revised: normative and accuracy data for seniors with heterogeneous educational level in Brazil. Int Psychogeriatr. 2017:29(8):134553. https://doi.org/10.1017/S1041610217000734.

10. Brasil. Conselho Nacional de Saúde. Resolução $n^{\circ}$ 466 de 12 de dezembro de 2012. Icapturado 2019 Out 24l. Disponivel em: http://bvsms.saude.gov.br/bvs/ saudelegis/cns/2013/res0466_12_12_2012.html.

11. Bowen CE, Kessler EM, Segler J. Dementia worry in middle-aged and older adults in Germany: sociodemographic, health-related and psychological correlates. Eur J Ageing. 2018 Feb;28;16(1):39-52. https://doi. org/10.1007/s10433-018-0462-7.

12. Bottino CM, Azevedo D Jr, Tatsch M, Hototian SR, Moscoso MA, Folquitto J, et al. Estimate of dementia prevalence in a community sample from São Paulo, Brazil. Dement Geriatr Cogn Disord. 2008;26(4):291-9. https://doi.org/10.1159/000161053.

13. Perera G, Pedersen L, Ansel D, Alexander M, Arrighi HM, Avillach P, Foskett N, Gini R, Gordon MF, Gungabissoon U, Mayer MA, Novak G, Rijnbeek P, Trifirò G, van der Lei J, Visser PJ, Stewart R. Dementia prevalence and incidence in a federation of European Electronic Health Record databases: The European Medical Informatics Framework resource. Alzheimers Dement. 2018 Feb;14(2):130-9. https://doi.org/10.1016/j.jalz.2017.06.2270.
14. Souza RKM, Barboza AF, Gasperin G, Garcia HDBP, Barcellos PM, Nisihara R. Prevalência de Demência em pacientes atendidos em um hospital privado no sul do Brasil. Einstein (São Paulo). 2020;18:1-7

15. de Miranda LFJR, Matoso RO, Rodrigues MV, de Lima TOL, Nascimento AF, Carvalho FC, Moreira DRM, Fernandes JC, de Paula JJ, Magno LAV, Caramelli P, de Moraes EN. Factors influencing possible delay in the diagnosis of Alzheimer's disease Findings from a tertiary Public University Hospital. Dement Neuropsychol. 2011;5(4):328-331. https://doi.org/10.1590/ S1980-57642011DN05040011.

16. Bottino CM, Azevedo D Jr, Tatsch M, Hototian SR, Moscoso MA, Folquitto J, et al. Estimate of dementia prevalence in a community sample from São Paulo, Brazil. Dement Geriatr Cogn Disord. 2008;26(4):291-9. https://doi.org/10.1159/000161053.

17. Correa Ribeiro PC, de Souza Lopes C, Lourenço RA. Prevalence of dementia in elderly clients of a private health care plan: a study of the FIBRA-RJ, Brazil. Dement Geriatr Cogn Disord. 2013:35(1-2):77-86. https:// doi.org/10.1159/000345984.

18. Darwish H, Farran N, Assaad S, Chaaya M. Cognitive Reserve Factors in a Developing Country: Education and Occupational Attainment Lower the Risk of Dementia in a Sample of Lebanese Older Adults Front. Front Aging Neurosci. 2018;10:277. https://doi.org/10.3389/fnagi.2018.00277.

19. Sobral M, Pestana MH, Paul C. The importance of quantification of cognitive reserve. Rev port enferm saúde mental. 2014:12:51-8.

20. Farina M, Paloski LH, Oliveira CR, Argimon IIL, Irigaray TQ. Cognitive Reserve in Elderly and Its Connection with Cognitive Performance: A Systematic Review. Ageing Int. 2018;43(4):496-507. https://doi.org/10.1007/ s12126-017-9295-5.

21. Singh-Manoux A, Dugravot A, Fournier A, Abell J, Ebmeier K, Kivimäki M, Sabia S. Trajectories of Depressive Symptoms Before Diagnosis of Dementia: A 28-Year Follow-up Study. JAMA Psychiatry. 2017 Jul 1;74(7):712718. https://doi.org/10.1001/jamapsychiatry.2017.0660.

22. Alexopoulos GS, Meyers BS, Young RC, Mattis S, Kakuma T. The course of geriatric depression with "reversible dementia": a controlled study. Am J Psychiatry. 1993:150(11):1693-9. https://doi.org/10.1176/ajp.150.11.1693.

23. Enache D, Winblad B, Aarsland D. Depression in dementia: epidemiology, mechanisms, and treatment. Curr Opin Psychiatry 2011;24:461-72. https://doi.org/10.1097/ YCO.0b013e32834bbgd4.

24. Reijnders JS, Ehrt U, Weber WE, Aarsland D, Leentjens AF. A systematic review of prevalence studies of depression in Parkinson's disease. Mov Disord. 2008:23:183-9. https://doi.org/10.1002/mds.21803.

25. Beckert M, Loureiro F, Menta C, Mello EF, Nogueira EL, von Gunten A, Gomes I. Performance of low-educated elders with depression on Addenbrooke's Cognitive Examination-Revised (ace-r) test. Dement Neuropsychol. 2016 Jan-Mar;10(1):19-25. https://doi. org/10.1590/s1980-57642016dn10100004. 
26. Vismari L, Alves GJ, Palermo-Neto J. Depressão, antidepressivos e sistema imune: um novo olhar sobre um velho problema. Rev Psiquiatr Clin. 2008;35(5):196-204. https://doi.org/10.1590/S0101-60832008000500004.

27. American Psychiatry Association. Diagnostic and Statistical Manual of Mental disorders - DSM-5. 5th.ed. Washington: American Psychiatric Association; 2013. https://doi.org/10.1176/appi.books.9780890425596.

28. Rock P, Roiser J, Riedel W, Blackwell A. Cognitive impairment in depression: A systematic review and meta-analysis. Psychol Med. 2014;44(10):2029-40. https:// doi.org/10.1017/S0033291713002535.

\section{Bruno Lodi}

Especialista em Psiquiatria pela Pontificia Universidade Católica do Rio Grande do Sul (PUCRS), em Porto Alegre, RS, Brasil.

\section{Paula Engroff}

Doutora em Gerontologia Biomédica pela Pontifícia Universidade Católica do Rio Grande do Sul (PUCRS), em Porto Alegre, RS, Brasil.

\section{Francisco Pascoal Júnior}

Doutor em Gerontologia Biomédica pela Pontificia Universidade Católica do Rio Grande do Sul (PUCRS), em Porto Alegre, RS, Brasil.

\section{Guilherme Marcos Nogueira}

Mestre em Gerontologia Biomédica pela Pontificia Universidade Católica do Rio Grande do Sul (PUCRS), em Porto Alegre, RS, Brasil.

\section{Vanessa Sgnaolin}

Doutora em Gerontologia Biomédica pela Pontifícia Universidade Católica do Rio Grande do Sul (PUCRS) em Porto Alegre, RS, Brasil.

\section{Alfredo Cataldo Neto}

Doutor em Medicina e Ciências da Saúde pela Pontifícia Universidade Católica do Rio Grande do Sul (PUCRS), em Porto Alegre, RS, Brasil; professor da Pontificia Universidade Católica do Rio Grande do Sul (PUCRS), em Porto Alegre, RS, Brasil.

\section{Endereço para correspondência}

Vanessa Sgnaolin

Pontificia Universidade Católica do Rio Grande do Sul Av. Ipiranga, 6681, Prédio 81, 7 andar, sala 703

Partenon, 90619-900

Porto Alegre, RS, Brasil 